\title{
Electroretinography as a Prognostic Indicator or Neovascularisationin CRVO
}

\author{
A. J. MORRELL*, D. A. THOMPSON, $\dagger$ J. M. GIBSON*, E. E. KRITZINGER*, \\ N. DRASDO $\dagger$ \\ Birmingham
}

\begin{abstract}
Summary
A prospective study was carried out to compare the efficacy of electroretinography, fundus fluorescein angiography and clinical examination in identifying those at risk of rubeotic glaucoma following central retinal vein occlusion (CRVO). Our preliminary observations are described. The findings suggest a complementary role for electroretinography in the management of CRVO. Particularly significant were interocular differences in $30 \mathrm{~Hz}$ flicker latency, the ability to elicit pattern ERGs and the ratio of scotopic and photopic a:b wave amplitudes. Of the clinical measures the depth of the relative afferent pupil defect was a sensitive indicator of rubeosis. Factors of lesser statistical prognostic value included poor visual acuity and the extent of deep retinal haemorrhages. Fundus fluorescein angiography in this study had limited value in predicting those patients at risk of rubeosis.
\end{abstract}

The development of neovascularisation and secondary glaucoma is a significant complication of central retinal vein occlusion (CRVO) occurring in $17-33 \%$ of patients. ${ }^{1,2,3}$ It is essential that those patients who are at risk of developing new vessels are identified at an early stage as prophylactic laser panretinal photocoagulation has been shown to be effective in eliminating the neovascular response. ${ }^{4,5}$

It has been suggested that the stimulus for new vessel growth is a vasoproliferative factor released by ischaemic retina. ${ }^{6}$ At present clinical and fluorescein angiographic features are used to determine the degree of retinal ischaemia present and to predict those eyes which will develop neovascularisation. However, these methods lack specificity, and result in the prophylactic treatment of a much larger number of patients than would be expected to develop complications. ${ }^{7}$ This largely reflects the limitations of fundus fluorescein angiography: assessment of the degree of retinal non-perfusion on the angiogram can be technically difficult due to factors such as small pupil size, media opacities and the presence of extensive deep retinal haemorrhages obscuring the retinal circulation. Even with good quality angiograms inconsistencies of interpretation occur amongst experienced observers. $^{8}$

New vessel growth in venous occlusion is considered to be a response to hypoxia rather than retinal cell death. ${ }^{1,2,6}$ Fluorescein angiography identifies under-perfused areas of retina, but these may be either hypoxic or infarcted retina. It is possible that the electroretinogram (ERG), which can distinguish decreased retinal sensitivity from non-functioning retina, may be a better means of pre-

From: *Birmingham and Midland Eye Hospital, Birmingham UK, †Vision Sciences, Aston University, Birmingham, UK.

Correspondence to: A. J. Morrell, Birmingham and Midland Eye Hospital, Church Street, Birmingham B3 2NS. 
dicting neo-vascularisation. The $a-$ and $b-$ wave components of the ERG respectively reflect the functional integrity of the receptoral and inner nuclear layers, which in turn depend upon differing blood supplies. Some earlier studies of the ERG in CRVO have demonstrated that patients with non-perfused CRVO, had reduced b/a wave amplitude ratios. ${ }^{9,10}$ It is possible this ratio may be a sensitive index of perfusion from the retinal circulation, because of the differential locus of generation of the two waves: the b-wave being the more proximal. However, these findings gave only an approximate indication of subsequent neovascularisation. Other recent reports have suggested ERG latencies, which reflect retinal sensitivity, may be useful additional indicators of new vessel formation. ${ }^{11,12,13}$

The ERG evoked by a pattern stimulus (PERG) is considered to have an even more proximal retinal origin than the scotopic b-wave. ${ }^{14.15}$ It might therefore register signs of a compromised retinal circulation earlier than a flash evoked ERG.

The PERG is a localised test of central retinal function and would not reflect the total extent of hypoxia present in the retina. However Magargal et al. ${ }^{16}$ has suggested that broad zones of capillary nonperfusion extend from the posterior pole to the retinal periphery. Should this be the case then the PERG, by sampling the posterior pole region, might be an index of the total amount of retinal ischaemia and thus be related to the likelihood of subsequent neovascularisation.

The aim of our study was to compare the efficacy of both flash and pattern electroretinography with currently employed clinical and fluorescein angiographic methods as a prognostic indicator of neovascularisation in patients with recent onset CRVO.

\section{Patients and Methods}

\section{Patients}

Forty-three consecutive patients, who presented to the Birmingham and Midland Eye Hospital casualty department between December 1988-Decmber 1989 with uniocular CRVO, were recruited. Fellow eyes were examined and patients with bilateral ocular involvement or other major retinal vas- cular disorders were excluded. The mean age of the group was 61 years, range 29-87 years. All patients were seen in the Medical Ophthalmology Clinic within three weeks of presentation for clinical assessment and fundus fluorescein angiography. Electroetinography was carried out with 7-12. days of (usually prior to) the fundus fluorescein angiogram. Informed consent was obtained from all patients entered into the study: (one patient refused consent for fluorescein angiography due to a fear of injections).

\section{Methods}

\section{Clinical Assessment}

Examination, undertaken by an observer who was unaware of the ERG results, included slit-lamp microscopy, intraocular pressure measurement, gonioscopy, fundoscopy and colour fundus photography. The following features were recorded: visual acuity," the number of fundal cotton wool spots, the extent of deep retinal haemorrhages, the presence of macular oedema, retino-ciliary collateral vessels and iris or retinal new vessels. If present a relative afferent defect was quantified using neutral density filters. ${ }^{17}$

\section{Fundus Fluorescein Angiography}

Fluorescein angiography was performed with a $50^{\circ}$ fundus camera taking sequential views of the posterior pole and the mid-periphery of the retina. Significant ischaemia was defined as greater than $50 \%$ capillary non-perfusion of the total retinal visible on the fluorescein angiogram and/or disruption of the perifoveal arcade.

\section{Electroretinography}

PERG recordings are demanding for the patient, requiring steady fixation and focus. The total ERG protocol was therefore designed to minimise patient chair time: a recording session including flash and pattern ERGs typically took 1-1.5 hours. Expendable DTL fibre corneal electrodes in detachable holders $^{18}$ were referred to EEG cup electrodes positioned on the ipsilateral temporal bone. Binocularly evoked responses were averaged on a Nicolet Pathfinder and stored on magnetic media for subsequent analysis.

\section{Flash ERGs}

Opaque diffusing goggles were used to distri- 
bute evenly the light flashes emitted by a Grass Strobe. Photopic ERGs were recorded to flash intensities $2,4,8$ and $16.10-20$ responses were averaged between a bandpass of $0.5-250 \mathrm{~Hz}$. Oscillatory potentials were filtered offline, between $150-250 \mathrm{~Hz}$, from the photopic response to flash intensity 16 . A $30 \mathrm{~Hz}$ flicker response was elicited by flash intensity 8 and 20 averages recorded between a bandpass of $0.5-70 \mathrm{~Hz}$. Single flash scotopic ERGs were obtained after a minimum of 15 minutes dark adaptation to flash intensity 8 . [Two patients were unable to tolerate the dark adaptation period because of claustrophobia even though the subject room was sizeable and a bowl stimulus was not used].

\section{Pattern ERGs}

Four black and white chequerboard patterns were used with decreasing effective retinal contrast. They were back-projected onto a circular screen of mean luminance $250 \mathrm{~cd} / \mathrm{m}^{2}$. Stimulus contrast was $75 \%$. The patterns were presented in order of decreasing retinal contrast:

The first two patterns were counterphased at $4 \mathrm{~Hz}$ :

1. A chequerboard subtending $5^{\circ} 30^{\prime}$

2. A chequerboard subtending $30^{\prime}$

The remaining two patterns were presented in onset/offset mode, appearing for $125 \mathrm{msec}$ and then disappearing into a background of mean luminance for $125 \mathrm{msec}$ :

3. A chequerboard subtending $5^{\circ} 30^{\prime}$

4. A radial chequerboard of 112 elements arranged around a central circle of $2^{\circ} 30^{\prime}$ containing a uniform chequerboard of 3'.

\section{Results}

Four patients were considered to be at risk of neovascularisation on the basis of their clinical and fundus fluorescein angiographic findings at their initial MOC visit and received immediate prophylactic laser photocoagulation. Two of the four were treated before electroretinography could be performed and were excluded from this study. For statistical purposes the remaining two patients who underwent ERGs before treatment were considered to be part of the non-rubeotic group. The final sample size was therefore 41 patients.
Patients were followed in clinic for a mean of 7.8 months (range 4-14 months), with the exception of one patient who died at two months. Eight of 41 patients developed rubeosis iridis within a mean of 10.4 weeks (range 3-20 weeks) from the estimated date of onset of CRVO. Three of these eight patients also developed new vessels on the disc. For subsequent analysis the patients were divided into rubeotic and non-rubeotic groups. The initial ERG data and clinical findings were compared across the two groups to find the most sensitive predictive factor of rubeosis.

\section{Clinical Assessment Results}

\section{Relative afferent pupil defect}

All patients were examined for the presence of a relative afferent pupil defect (RAPD). Twenty patients were found to exhibit a defect, including all eight rubeotic patients. The RAPD was quantified in 16 patients, but not in four of the patients who subsequently became rubeotic. The presence and magnitude of the RAPD was a sensitive method of differentiating the two groups of patients (Mann-Whitney test, $\mathrm{P}<0.0005)$.

\section{Visual acuity}

In order to include measures of PL, HM and $\mathrm{CF}$, visual acuities were grouped into three bands: $(<6 / 60),(6 / 60-6 / 18)$ and $(>6 / 18)$. As shown in Table I, poor visual acuity was found to be of significance in distinguishing the rubeotic group at the level $\mathrm{p}<0.025$ (Chisquare test). If the visual acuities were grouped into two bands: $<6 / 60$ and $6 / 60$ or better, then the significance level was found to increase to $-<0.015$.

\section{Retinal heamorrhages}

The extent - of deep retinal haemorrhages, graded $0-3$ according to the proportion of ret-

Table I Visual acuity. Poor visual acuity was of significance in predicting rubeosis at the level $p<0.025$ (Chi square test). Grouping the visual acuities into 2 bands: $<6 / 60$ and $6 / 60$ or better, increased the level of significance to $p<0.015$

\begin{tabular}{lrcccl}
\hline & $<6 / 60$ & $6 / 6 ;-6 / 18$ & $>6 / 18$ & \\
\hline Rubeotic & 5 & 1 & 2 & $=8$ \\
Non-rubeotic & 6 & 20 & 7 & $=33$ \\
Total & 11 & 21 & 9 & $=41$ \\
\hline
\end{tabular}


Table II Results of assessment by fundus fluorescein angiography. Three out of eight patients were identified as being ischaemic and therefore at risk of rubeosis by fluorescein angiography

\begin{tabular}{lccc}
\hline & \multicolumn{3}{c}{ Classification by fluorescein angiography } \\
& ischaemic & non-ischaemic & borderline \\
\hline Final outcome & 3 & 2 & 0 \\
Rubeotic & 2 & 0 & 0 \\
Prophylactic laser & 0 & 25 & 2 \\
Non-rubeotic & & & Total 34 \\
& & & \\
\hline
\end{tabular}

Table III Interocular difference in flicker ERG latency. The phase delay was significantly greater in the rubeotic group: Student t-test, $p<0.002$

\begin{tabular}{lccc}
\hline & Number of patients & $\begin{array}{c}\text { Interocular difference in } \\
\text { flicker latency }\end{array}$ & \\
\hline Rubeotic & 7 & $9.78 \mathrm{~ms}$ & $\mathrm{SD}=3.28$ \\
Non-rubeotic & 31 & $4.66 \mathrm{~ms}$ & $\mathrm{SD}=3.78$ \\
\hline
\end{tabular}

ina involved $(0=$ absent, $1=$ mild, $2=$ moderate, $3=$ severe), was found to be of borderline significance in distinguishing the two groups (Mann-Whitney significant at $\mathrm{p}<0.049)$.

\section{Cotton wool spots}

Statistically the number of cotton wool spots, grouped (0-3), (4-7), (8-11) and (12-15), was a poor indicator of subsequent rubeosis (Mann-Whitney $\mathrm{p}<0.13$ ).

The development of collateral vessels and the presence of macular oedema did not relate statistically to the subsequent development of rubeosis.

\section{Fundus Fluorescein Angiography Results}

Fundus fluorescein angiography was not undertaken in two patients: in one patient the procedure was contra-indicated because of severe asthma and in the other because of lack of consent. In five patients no assessment of the angiogram was possible: in three due to extensive retinal haemorrhages, in one due to an allergic reaction to the fluorescein and in another due to the confused state of the patient. In a further six patients the perifoveal arcade was obscured, however, it was possible to grade the degree of capillary non-perfusion as a percentage of the total retina visible on the angiogram. Table II shows that only three of the eight rubeotic patients were identified as being at risk by fluorescein angiography.

\section{Electroretinographic Results}

\section{Flash electroretinography}

The flash electroretinography data in the form of the amplitude ratios e.g. photopic and scotopic a:b -wave amplitude, b-wave latency and flicker phase difference between affected and unaffected eyes were statistically evaluated. The interocular difference in the flicker ERG latency was found to be the most significant distinguishing feature (see Table III) with the phase delay being greater in the rubeotic group (Student t-test, $\mathrm{p}<0.002$ ).

The amplitudes of the a- and b-waves of the scotopic ERG were measured and the ratio $a / b$ found. The difference between ratios of rubeotic and non-rubeotic eyes were compared using a Student t-test (Table IV) and found to be significant, $\mathrm{p}<0.001$. However, the standard deviation was greater than the mean value for each group and therefore this measure is not as specific as the interocular difference in flicker latency in distinguishing the at risk group.

\section{Pattern electroretinography}

The PERGs to the largest stimuli were absent for the majority of rubeotic patients: thus amplitude measures between the sub-groups could not be compared. The PERG stimuli was therefore graded 1-4 according to their effective retinal contrast, and a record made of the number of different patterns to which a response could be elicited. Figure 1 illustrates 
Table IV Interocular difference scotopic $a / b$ wave amplitude ratio. The mean interocular difference in the scotopic $a / b$ wave amplitude was found to be $a$ significant indicator of rubeosis (student t-test $p<0.001$ )

\begin{tabular}{lrcl}
\hline & pt nos & mean diff \\
\hline Rubeotic & 7 & 0.311 & SD $=0.354$ \\
Non-rubeotic & 29 & 0.003 & SD $=0.137$ \\
\hline
\end{tabular}

that this number was found to be a sensitive indicator of the likelihood of neovascularisation (Table V, Mann-Whitney test $\mathrm{p}<0.0034)$.

\section{Discussion}

The results from this prospective study support the findings of previous authors on the useful role of electroretinography in identifying patients at risk of developing rubeosis, and for the first time demonstrates a role for the pattern electroretinogram in assessing these patients. Although amplitude measures could not be compared between rubeotic and nonrubeotic patients, the number of different patterns to which a PERG could be recorded was found to be a sensitive prognostic indicator of neovascularisation.

Analysis of the flash ERG data demonstrates alterations of both response amplitudes and latencies in the affected eyes. To identify those eyes at risk of rubeosis some authors have reported greater discrimination

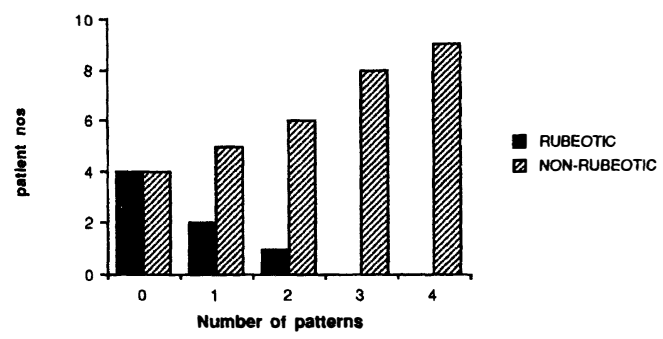

Fig. 1. Perg data. 4 patterns of decreasing effective retinal contrast were used to elicit the PERG's. The number of patterns to which a PERG could be recorded was a significant prognostic indicator of rubeosis at the $p<0.0034$ level (Mann-Whitney test). when amplitude measures are compared ${ }^{9,10.13}$ whilst others have found the latency results more accurately distinguish the two groups of eyes. ${ }^{11,12}$

This study has shown that mean interocular differences in implicit response time and in both scotopic and photopic a:b amplitude ratios to be significant indicators of subsequent rubeosis. Of these, interocular flicker latency difference is the most meaningful discriminant measure: a difference of six milliseconds or greater would have correctly identified all eight patients as being at risk of developing rubeosis with a false positive rate of $2 \%$, i.e. nine of 43 patients, (of these one received prophylactic laser and one died within two months and has been considered in the non-rubeotic group). In comparison an interocular difference in $a: b$ scotopic ratio of 0.3 or greater would correctly identify only four of the rubeotic group but included only one false positive.

The clinical data indicated the depth of the relative afferent pupil defect was the most significant clinical criteria. Servais et al. ${ }^{18}$ reported $91 \%$ of ischaemic CRVO patients had a relative afferent pupil defect of $1.2 \mathrm{log}$ units or more. Within the limits of the small sample size our data showed that, taking a relative afferent pupil defect of $1.8 \log$ units or more as the threshold value, all rubeotic patients would have been identified with only one false positive case. The number of cotton wool spots present at initial examination was found to be a poor indicator of subsequent rubeosis whilst retinal haemorrhages and visual acuity were of marginal significance in predicting outcome. This data has to be interpreted with caution because of the small sample size involved. It is possible that factors of apparent marginal significance may assume greater significance if a larger sample size was involved.

Fluorescein angiography has been used extensively to assess the degree of retinal ischaemia following vein occlusion, but is an

Statistical analyses were performed with the MINITAB and GLIM packages. This comprised the Chi-squared test for categorical data and the student t-test or Mann-Whitney U test for continuous data group comparisons. The nonparametric test being enlarged if the data were non-symmetrically distributed or inexact (e.g. visual acuities: $\mathrm{CF}<\mathrm{PL}<\mathrm{NPL}$ ). 
Table $\mathbf{V}$ Perg data. The number of different patterns to which a response could be elicited was a significant prognostic indicator of rubeosis. Mann-Whitney test $p<0.0034$.

number of patients median number of patterns

$\begin{array}{rr}7 & 0.0 \\ 32 & 2.0\end{array}$

2.0
Rubeotic group

Non-rubeotic group invasive procedure with recognised problems of standardising the assessment. The experience of this study confirmed the difficulties encountered by other authors with either failure to obtain an angiogram in every patient or of technical problems of interpretation e.g. due to masking by retinal haemorrhage.${ }^{19}$ For such reasons only three of eight rubeotic patients in this sample group were successfully identified as being at risk by fluorescein angiography.

Although criteria such as depth of RAPD and ERG measures are sensitive indicators of rubeosis, the false positive rate using multiple discriminant analysis combining both clinical and ERG data would improve the specificity. We are currently examining its possibility.

\section{Conclusions}

In this preliminary study significant distinguishing features with high predictive values for the development of rubeosis iridis are:-

(1) the interocular flicker latency difference

(2) the ability to elicit pattern ERGs

(3) the depth of the relative afferent pupil defect

(4) the ratio of scotopic and photopic a:b wave amplitude ratios

Factors of lesser statistical prognostic value included:

(5) a visual acuity less than $6 / 60$

(6) the extent of deep retinal haemorrhages

(7) fundus fluorescein angiography

(8) the number of cotton wool spots.

We are grateful to Mr. Peter Francois, Senior Lecturer, Dept of Vision Sciences, Aston University and Mr. David Shaw, Research Assistant, University of Birmingham, Department of Ophthalmology for the statistical analysis performed on the data of this study. We would like to thank Dr. Lesley Jones and Miss Vivaca Vann Der Vliet of the Clinical Neurophysiology Unit, Dept of Vision Sciences, Aston University for their technical assistance with the electroretinography and Mr. Ivan Bradley, Birmingham and Midland Eye Hospital, for his assistance with the photography.

KEY wORDS: Central Retinal Vein Occlusion-Flash
electroretinography-Pattern electroretinographyFluorescein angiography-Rubeosis.

References

${ }^{1}$ Magargal LE, Brown GC, Augsburger JJ, Parrish RK: Neovascular glaucoma following central retinal vein obstruction. Ophthalmology 1981; 88: 1095-100.

${ }^{2}$ Hayreh SS: Classification of Central Retinal Vein Occlusion. Ophthalmology 1983; 90: 458-72.

${ }^{3}$ Laatikainen L and Kohner EM: Fluorescein angiography and its prognostic significance in retinal vein occlusion. Br J Ophthalmol 1976; 60: 411-18.

${ }^{4}$ May DR, Klein ML, Peyman GA, Rauchand M: Xenon arc panretinal photocoagulation for central retinal vein occlusion: a randomised prospective study. Br J Ophthalmol 1979; 63: 725-34.

${ }^{5}$ Laatkainen L: Preliminary report on effect of retinal panphotocoagulation on rubeosis iridis and neovascular glaucoma. Br J Ophthalmol 1977; 61: .278-84.

${ }^{6} \mathrm{Katz}$ A: Clinical and experimental studies on retinal neovascularisation. Am J Ophthalmol 1982; 94: 715-43.

${ }^{7}$ Magargal LE, Brown GC, Augsburger JJ, Donoso LA: Efficacy of panretinal photocoagulation in preventing neovascular glaucoma following ischaemic central retinal vein occlusion. Ophthalmology 1982; 89: 780-4.

${ }^{8}$ Welch JC and Augsburger JJ: Assessment of angiographic retinal capillary non-perfusion in central retinal vein occlusion. Am J Ophthalmol 1987; 106: $353-6$.

${ }^{9}$ Sabates R, Hirose T, McMeel JW: Electroretinography in the prognosis and classification of central retinal vein occlusion. Arch Ophthalmol, 1983; 101: 232-5.

${ }^{10}$ Barber C, Galloway N, Reacher M, Salem H: The role of the electroretinogram in the management of central retinal vein occlusion. Doc Ophthalmol Proc Series 1984; 40: 149-59.

${ }^{11}$ Johnson MA, Marcus S, Elman MJ, McPhee TJ: Neovascularisation in central retinal vein occlusion: electroretinographic findings. Arch Ophthalmol 1988; 106: 348-52.

${ }^{12}$ Kaye SB and Harding SP: Early electroretinography in unilateral central retinal vein occlusion as a predictor of rubeosis iridis. Arch Ophthalmol 1988; 106: 353-6.

${ }^{13}$ Breton ME, Quinn GE, Keene S, Dahmen JC, Brucker AJ: Electroretinogram parameters at presentation as predictors of rubeosis in central retinal vein occlusion patients. Ophthalmology 1989; 96: 1343-52.

${ }^{14}$ Lawill T: The bar pattern electroretinogram. Doc Ophthalmol 1984; 40: 1-10. 
${ }^{15}$ Holder GE: Significance of abnormal pattern electroretinography in anterior visual pathway dysfunction. Br J Ophthalmol 1987; 71: 166-71.

${ }^{16}$ Magargal EL, Donoso LA, Sanborn GE: Retinal ischaemia and risk of neovascularisation following central retinal vein occlusion. Ophthalmology 1982; 89: 1241-5.

${ }^{17}$ Thompson DA, Corbett JJ, Cox TA: How to measure the relative afferent pupil defect. Surv Ophthalmol 1981: 26: 39-42.
${ }^{18}$ Thompson DA and Drasdo N: An improved method for using the DTL fibre in electroretinography. Ophthal, Physiol Opt 1987; 7: 315-19.

${ }^{19}$ Servais GE, Thompson HS, Hayreh SS: Relative afferent pupil defect in central retinal vein occlusion Ophthalmology 1986; 93: 301-3.

${ }^{20}$ Minturn J and Brown GC: Progression of nonischaemic central retinal vein obstruction to the ischaemic type. Ophthalmology 1986; 93: 1158-62. 